\title{
Color Doppler Evaluation of Carotid Arteries in Stroke Patients: A Study Conducted in A rural Tertiary Care Medical College Hospital in South india.
}

\author{
Dr.Madhavi Chamarthi.,MD ${ }^{1}$,Dr.Lava Kumar B,MD²,Dr.Nirusha $\mathrm{R}^{3}$, \\ Dr.Pravallika I ${ }^{4}$,Dr.G.S.Kejriwal ${ }^{5}$, \\ ${ }^{1}$ Associate professor of Radiology Department of radio-diagnosis,Maharajah's institute of medical sciences, \\ Nellimarla, A.P. \\ ${ }^{2}$ Senior Resident, Department of radio-diagnosis Great Eastern medical school and hospital, Srikakulam, A.P. \\ ${ }^{3}$ Post graduate(M.D., Final year), Department of Radio-diagnosis Maharajah's institute of medical sciences, \\ Nellimarla, A.P. \\ ${ }^{4}$ Post graduate(M.D., first year), Department of Radio-diagnosis Maharajah's institute of medical sciences, \\ Nellimarla, A.P. \\ ${ }^{5}$ Head of the department, department of radio diagnosis, Maharajahs institute of medical seciences, \\ vizianagaram
}

\begin{abstract}
Aims and Objectives: Cerebral ischemic stroke is life-threatening and debilitating neurological disease, it is the third leading cause of death in the world. Studies have shown that there is a close relationship between carotid artery stenosis and ischemic cerebral vascular disease. Doppler study is valuable to assess the cause, location, extent and severity of carotid artery disease. The gray scale imaging provides information about the location. By combining the gray scale findings with colour Doppler characteristics, severity of stenosis is obtained. This study is done to assess the carotid arteries with the help of colour Doppler sonography and to correlate with CT findings of cerebrovascular accidents. There has been a recent proliferation of methods of assessing the extracranial circulation, including non-invasive techniques, such as the carotid Doppler ultrasound examination. Asymptomatic cerebral emboli can be detected using carotid Doppler ultrasound. These embolic signals have potential as a marker of stroke risk.

Materials and Methods: The prospective study was carried out on 75 patients using Philips HD7XE ultrasound machine over a period of 12 months in the Radiology department of Maharjah's Institute of Medical Sciences and correlated with Ge Dual Slice Ct Scan Unit. The data gathered included peak systolic velocity of common carotid artery (CCA) and internal carotid artery (ICA), velocity ratios between CCA and ICA and plaque characteristics as seen on real-time imaging.

Results: The highest incidence of stroke was found in the male population in the age group of 60-69 years. Hypertension followed by smoking were considered as potent risk factors. Most of the plaques were located at the bifurcation and majority of them were echogenic.Out of 75 patients, 16 patients showed significant stenosis (>60\%). Atherosclerotic plaques were seen in 57 patients (76\%).

Conclusion: Colour Doppler examination is an economic, safe, reproducible, and less time-consuming method of demonstrating the cause of cerebrovascular insufficiency in extracranial carotid artery system.
\end{abstract}

Keywords: Atherosclerotic plaque, colour Doppler sonography, common carotid artery, internal carotid artery, peak systolic velocity, peak systolic velocity ratio,stenosis

\section{Introduction}

Cerebrovascular accident or stroke is the sudden onset of focal neurologic deficit from a vascular mechanism. It is one of the major causes leading to death after malignancy and cardiovascular pathologies, atherosclerosis of cranial vessels leading to cerebral infarction being the main culprit. Greater risk of stroke is associated among patients with comorbidities like diabetes, asymptomatic lesions, higher degree of carotid artery stenosis or a combination of these parameters ${ }^{[1,2]}$. Colour Doppler study of carotid artery forms an essential task of assessing extra-cranial insufficiency. A non-invasive, economical screening tool is required to differentiate haemodynamically stable patients from those with higher degrees of stenosis. Duplex sonography combines high resolution imaging and Doppler spectrum analysis to yield effective means of detecting and assessing carotid disease, which has largely replaced angiography for suspected extra-cranial carotid atherosclerosis ${ }^{[3,4]}$.

Timely atherectomies have been documented to prevent many strokes, hence this necessitates to evaluate carotid artery system. Carotid angiography is considered a gold standard for evaluating severity of 
carotid disease, but certain drawbacks like invasive procedure and cost effectiveness limits its use. Besides estimating the degree of stenosis, greatest advantage of sonography lies in ability to characterize the plaque and identify plaques with a higher risk of embolization. With these parameters in mind, this study was carried out to assess the role of duplex sonography in determining carotid artery pathology, which lead to stroke.

\section{Aims \& Objectives}

To study the role of B mode sonography in carotid artery disease.

To assess carotid artery through colour Doppler.

To correlate the findings on duplex sonography with computed tomography images of brain in CVA patients.

\section{Materials And Methods}

This is a prospective study done over a period of 12 months on 75 patients of cerebrovascular insufficiency who presented to the department of Radio-Diagnosis in MIMS Hospital, mainly referred from departments of general medicine and emergency medicine.

\section{Inclusion criteria:}

Patients who were refereed to CT scan of brain for either of the symptoms -hemiparesis, hemiplegia, monoparesis, TIA,hemisensory loss, aphasia and on axial CT showed findings of infarct in territories of branches of internal carotid artery were subjected to carotid Doppler sonography.

\section{Exclusion criteria:}

Patients with an evidence of infarcts in vertebro-basilar territory, intracerebral haemorrhage, neoplasms and other causes of stroke were excluded.

\section{Equipment used:}

Doppler study was conducted on Philips HD7XE ultrasound machine using high frequency $10 \mathrm{MHz}$ linear array transducer. CT correlation was done with GE DUAL SLICE CT SCAN unit.

\section{Carotid doppler examination:}

In supine position with head of the patient slightly extended and turned away from the site of examination, $10 \mathrm{MHz}$ frequency linear array transducer is used to trace common carotid, external carotid and internal carotid arteries. In transverse plane, Internal and external carotid arteries were assessed for their calibre, location and size of the plaque, severity of luminal narrowing and size of the residual lumen. In longitudinal plane, two positions - anterolateral and posterolateral are routinely imaged. The angle corrected velocity spectrum to assess high resistance pattern of external carotid artery and low resistance pattern of internal carotid artery is done. For each lesion, extent of the plaque, surface characteristics, the internal appearance and visible flow abnormalities are recorded.

IV. Statistical Analysis And Results

In our study of 75 cases, 49 were male (65.4\%) and 26 were female (34.6\%) with a sex ratio of 2:1.

\begin{tabular}{|l|l|l|}
\hline Males & 49 & $65.4 \%$ \\
\hline Females & 26 & $34.6 \%$ \\
\hline Total & 75 & $100 \%$ \\
\hline
\end{tabular}

Table 1: sex distribution of cases

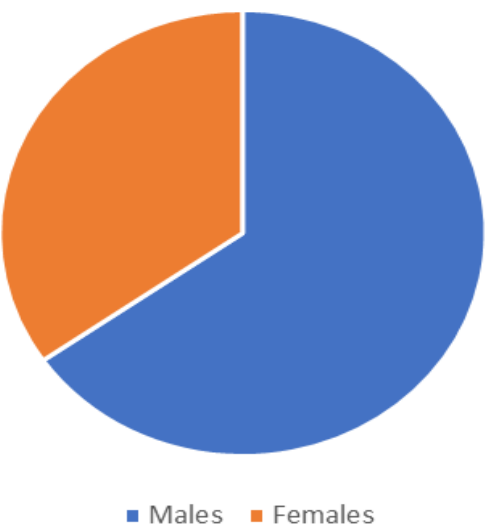

Figure 1: sex distribution of cases 
Color Doppler Evaluation of Carotid Arteries in Stroke Patients: A Study Conducted ...

Majority of patients belonged to age group $60-69$ years in both the genders.

\begin{tabular}{|l|l|l|l|l|}
\hline $\begin{array}{l}\text { Age Group } \\
\text { (In Years) }\end{array}$ & $\begin{array}{c}\text { Males } \\
\text { (Number) }\end{array}$ & $\begin{array}{l}\text { Males } \\
\text { (Percentage) }\end{array}$ & $\begin{array}{c}\text { Females } \\
\text { (Number) }\end{array}$ & $\begin{array}{c}\text { Females } \\
\text { (Percentage) }\end{array}$ \\
\hline$<40$ & 6 & $12.2 \%$ & 5 & $19.2 \%$ \\
\hline $40-49$ & 7 & $14.3 \%$ & 3 & $11.5 \%$ \\
\hline $50-59$ & 14 & $28.6 \%$ & 3 & $11.5 \%$ \\
\hline $60-69$ & 15 & $30.6 \%$ & 11 & $42.3 \%$ \\
\hline $70-79$ & 7 & $14.3 \%$ & 4 & $15.5 \%$ \\
\hline$>80$ & 0 & 0 & 0 & 0 \\
\hline
\end{tabular}

Table 2: age and sex distribution of cases.

Hypertension was found to be the common risk factor followed by smoking for > 10 years, hypercholesteremia and diabetes. Out of 75 patients, atheromatous plaque was found bilaterally in 29 patients (39\%), only on right side in 16 patients $(21 \%)$ and only on left side in 12 patients (16\%). Plaque was not observed at all in 18 patients $(24 \%)$.

\begin{tabular}{|l|l|l|}
\hline Site & Number Of Cases & Percentage $(\%)$ \\
\hline No Plaque & 18 & 24 \\
\hline Right & 16 & 21 \\
\hline Left & 12 & 16 \\
\hline Bilateral & 29 & 39 \\
\hline Total & 75 & 100 \\
\hline
\end{tabular}

Table 3: Distribution of atheromatous plaques

Out of 111 plaques, 34 were located in common carotid artery (18 on right and 16 on left), 21 in internal carotid artery (12 on the right and 9 on the left) and 56 at bifurcation (30 on right side and 26 on the left). Overall $50.4 \%$ of the plaques were located at bifurcation, 30.6\% in the common carotid artery and $19 \%$ in the internal carotid artery.

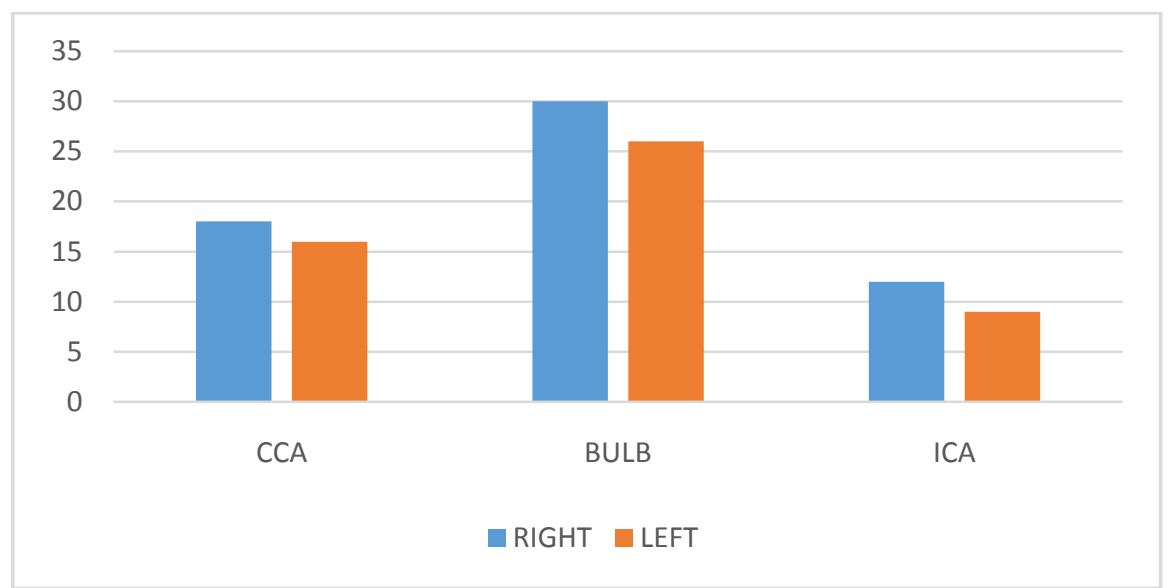

Figure 2: Distribution of atheromatous plaques according to site

About 111 plaques were evaluated in a total of 150 vessels examined.

Out of the 111 plaques, $34(31 \%)$ were calcified, $33(30 \%)$ were uniformly echogenic, $27(24 \%)$ were predominantly echogenic, $10(9 \%)$ were predominantly echolucent and 7(6\%) were uniformlecholucent.

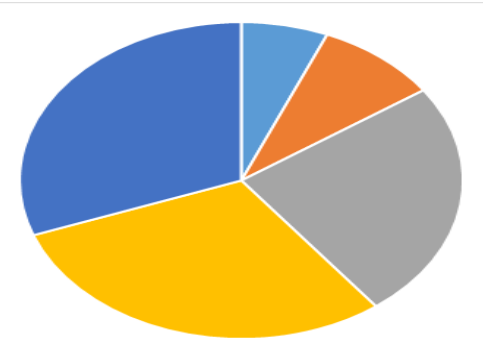

- Uniformly echolucent

uniformly echogenic

Figure 3: plaque characterisation 
Color Doppler Evaluation of Carotid Arteries in Stroke Patients: A Study Conducted ...

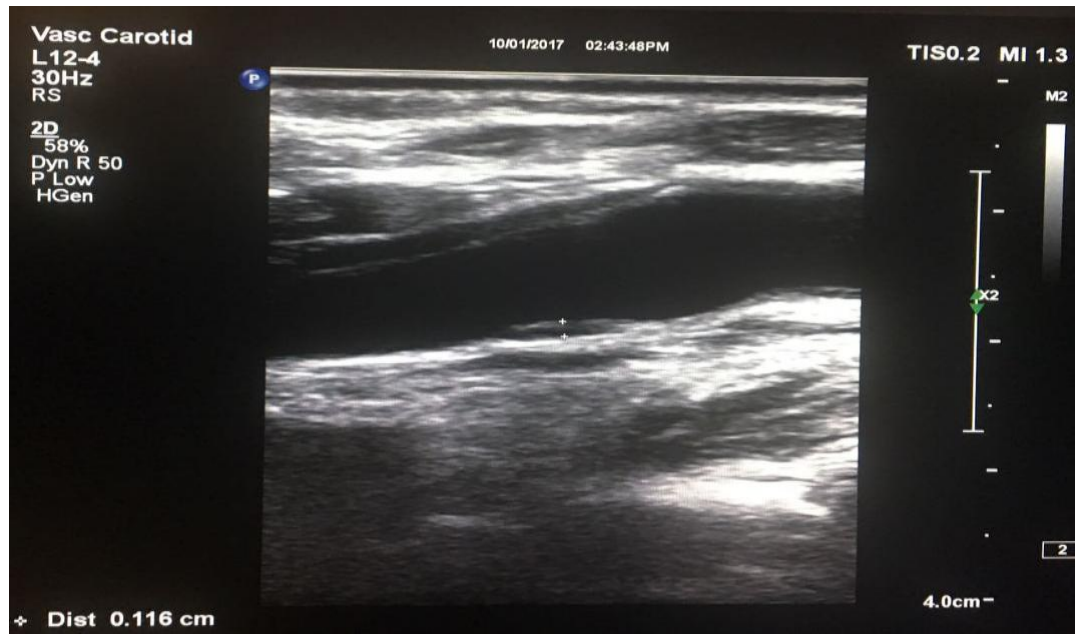

Figure 4: showing atheromatous plaque

Two vessels were completely occluded. Ulceration was detected on B mode in two of the plaques and on colour flow imaging in six plaques.

Out of 150 vessels examined, significant stenosis ( $>50 \%)$ was seen in $18(12 \%)$ vessels on colour flow imaging.

\begin{tabular}{|l|l|l|}
\hline Percentage Of Stenosis & $\begin{array}{l}\text { Number Of Vessels } \\
(\mathrm{N}=150)\end{array}$ & Percentage \\
\hline$<50$ & 132 & $88 \%$ \\
\hline $50-69$ & 10 & $6.7 \%$ \\
\hline $\begin{array}{l}>70 \text { But Less Than Near } \\
\text { Occlusion }\end{array}$ & 3 & $2 \%$ \\
\hline Near Occlusion & 3 & $2 \%$ \\
\hline Total Occlusion & 2 & $1.3 \%$ \\
\hline
\end{tabular}

Table 4: Distribution of cases based on percentage of stenosis

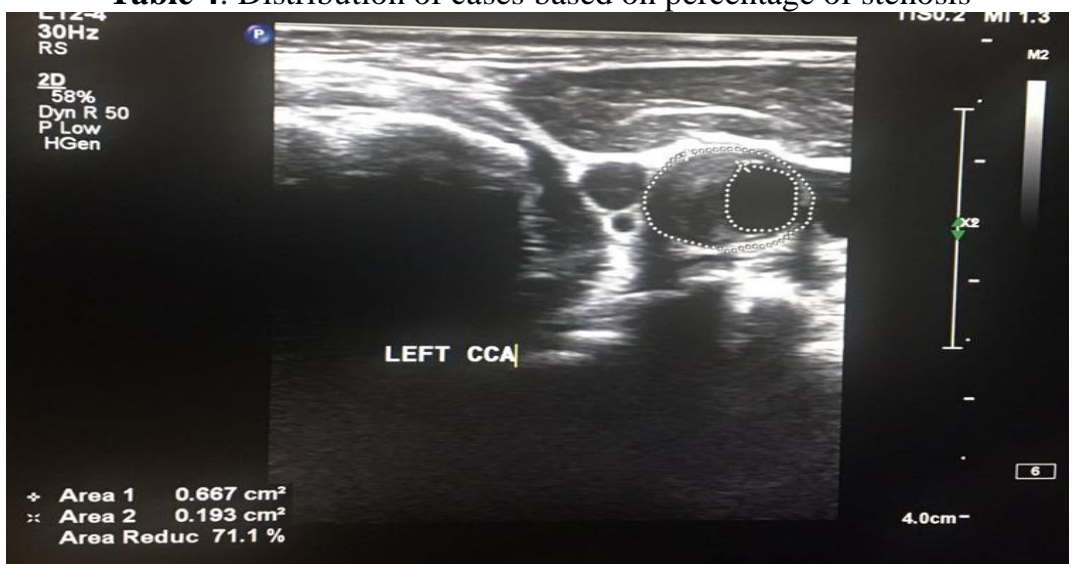

Figure 5: showing $71 \%$ luminal narrowing of left CCA

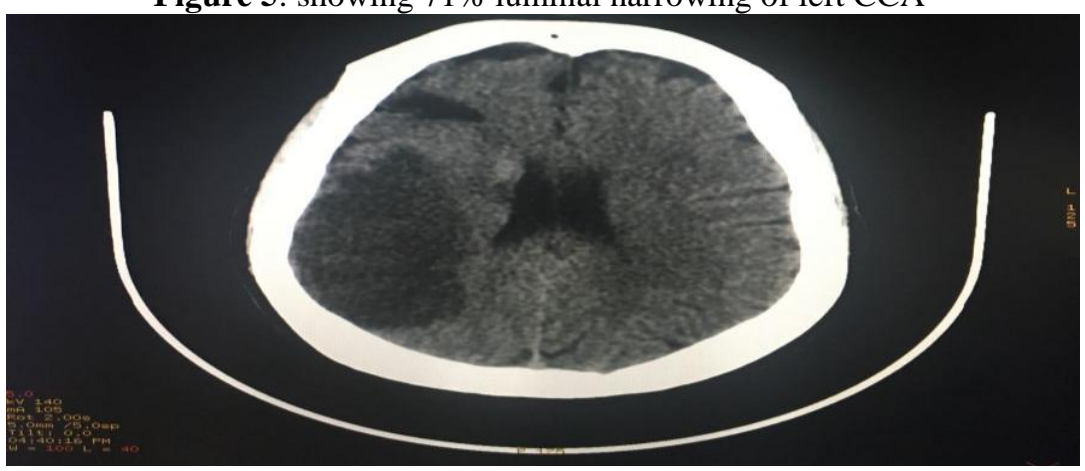

Figure 6 : showing axial CT correlation of left CCA luminal narrowing with acute infarct in right MCA territory. 
Color Doppler Evaluation of Carotid Arteries in Stroke Patients: A Study Conducted ...

In our study based on PSV ration of ICA/CCA, 18 vessels showed significant stenosis i.e, ratio >2.

\begin{tabular}{|l|l|l|}
\hline Psv Ratio Ica/Cca & $\begin{array}{l}\text { Number Of Vessels } \\
(\mathrm{N}=150)\end{array}$ & Percentage \\
\hline$<2.0$ & 132 & $88 \%$ \\
\hline 2.0 To 4.0 & 10 & $6.7 \%$ \\
\hline$>4.0$ & 3 & $2 \%$ \\
\hline
\end{tabular}

Table 5 : Distribution of cases based on PSV ratio of ICA/CCA

Out of 75 cases with infarcts, 34 patients $(45.3 \%)$ had infarcts on right, 34 on left and 7 patients $(9.4 \%)$ had bilateral infarcts.

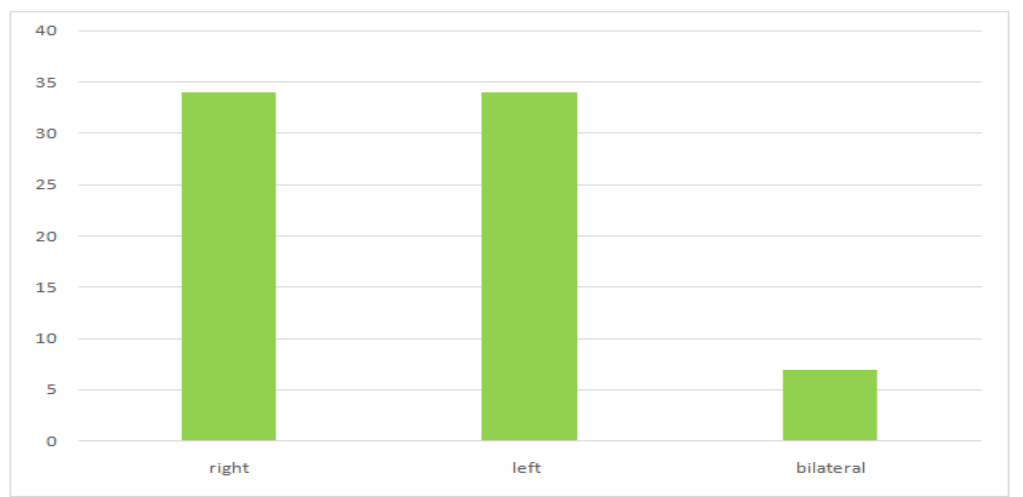

Figure 7: location of infarct

\section{Discussion}

Incidence of stroke has been estimated as about 794 per million population per year in the world and there has been marked reduction in the frequency of CVA with effective treatment of hypertension ${ }^{[5]}$. Cerebrovascular disease effects predominantly the older and middle age group, its prevalence increases with the increase in age ${ }^{[6]}$. Of all the CVA, $85 \%$ occur by occlusion of the extracranial or intracranial cerebral vessels, $10 \%$ due to primary intracerebral haemorrhage, and 5\% due to subarachnoid haemorrhage. It has been shown that $20 \%$ of strokes have been heralded by TIA. Asymptomatic carotid disease has annual stroke rate of $1.3 \%$, while symptomatic accounts for $15 \%$.

Cerebral ischemia is caused by reduction in blood flow that lasts for several seconds to few minutes. Cerebral infarction basically compromises 2 pathophysiology processes- one, loss in the supply of oxygen and glucose secondary to vascular occlusion and two, an array of changes in the cellular metabolism consequent on the collapse of energy producing processes within the disintegration of cell membranes. The effects of ischemia depend on degree and duration of cerebral insufficiency.

Cerebrovascular disease most commonly presents as acute focal stroke, but ischemic cerebral arterial disease may also present as gradual decline in intellectual function with or without limb deficits or gait disorders. Cerebral infarction is mostly due to thromboembolic disease secondary to atherosclerosis in major extra cranial arteries.

Prevalence of atherosclerosis increases with age. Wolf et al (1991) reported a mean age of 65.4 years in men and 66.1 years in females ${ }^{[7] .}$ In our study, out of 75 cases, 49 were males and 26 were females with mean age of prevalence in $60-69$ years which is also comparable to the study done by Paivansalo et al ${ }^{[8]}$.

We found in our study that hypertension was the commonest risk factor (78.7\%), which was analogous to the study done by Dhamija et al, who concluded hypertension was the most consistent risk factor and systolic blood pressure being more closely related to ischemic stroke than diastolic pressure ${ }^{[9]}$.

In our study, overall $50.4 \%$ of plaques were located at bifurcation, $30.6 \%$ in the common carotid artery and $19 \%$ in the internal carotid artery. This finding were similar to that observed by Rajagopal et al ${ }^{[10]}$ and SK Sethi et al ${ }^{[11]}$

In our present study, on B mode imaging ulceration was detected in two of the plaques (2\%) while oncolour flow imaging six $(5 \%)$ of the plaques showed evidence of ulceration. Steinke et al ${ }^{[12]}$ also found significant improvement in identification of ulcerated plaques with colour flow imaging over B mode scanning. Erickson et al ${ }^{[13]}$ and Steinke et al ${ }^{[12]}$ have calculated the percentage of stenosis by directly measuring the colour flow lumen at the site of maximum stenosis and then comparing it with total lumen of vessel itself. In our present study, we calculated degree of stenosis by directly measuring residual lumen at the site of maximum stenosis and comparing it with total lumen. At the site of maximal stenosis spectral analysis was done and degree of stenosis estimated using velocity criteria. 
According to Middleton et al ${ }^{[14]}$, the duplex imaging of complete carotid occlusion was based on the following observation- absence of arterial pulsation, lumen filled with echogenic material, subnormal vessels size and absence of Doppler flow signals or weak Doppler signals. In our study 2 cases of complete carotid stenosis were noted, which showed all the above characteristics of Doppler study.

All the patients having stroke, where carotid artery disease is the cause had carotid artery disease at expected site or were having bilateral involvement that is, all the patients having right sided stroke clinically had left sided infarct on CT and left extra cranial carotid artery disease and vice versa or had bilateral extra cranial carotid artery disease. These findings were similar to the observations of JS Jeng et al ${ }^{[15]}$

The main role of carotid Doppler examination in the carotid artery disease is detection of occlusive lesions in the vicinity of carotid bifurcation and internal carotid artery. The accurate diagnosis of critical stenosis is important because these are the patients who carry increased risk of cerebral infarction.

Angiography as the gold standard has amply been replaced by Duplex ultrasonography in most diagnostic circumstances. Positive experience with carotid duplex ultrasonography scanning unassisted by angiography in the setting of an impending surgical intervention has been embraced by a growing number of doctors. So, the present study was done to evaluate extra cranial carotid arterial system by carotid Doppler in the population who presented with cerebrovascular insufficiency

\section{Conclusion}

Patients with CVA where carotid artery was the cause, pathology was found on the expected site or bilaterally. The main causative lesion was atherosclerotic plaque in extra cranial carotid artery, with carotid bulb as the most common site being involved. B mode sonography is better in plaque characterization but hypoechoic, ulcerated plaques and thrombus were better confirmed on colour Doppler imaging. In our study we concluded that colour Doppler imaging was superior to B mode imaging in detecting high grade stenosis and differentiating total occlusion from near total occlusion. The present study highlights the importance of Doppler sonography in prevention of stroke through surveillance of atherogenesis in extracranial carotid system.

\section{References}

[1]. Haffner et al. The insulin resistance atherosclerosis study (IRAS). Circulation 2000;102(1):42-7

[2]. Gesis G et al Strong association between classic risk factors for atherosclerosis and increase IMT after population studies inducted in men and women of various groups-different constellations of risk factors Lancet 2000;1123-25.

[3]. Bluth E. Doppler sonography 'Uitrsound in new millennium”. ALUM Education Course.

[4]. Bluth E, Stavros A, Marich. Carotid Duplex sonography . A multicentre recommendation for standardized imaging and Doppler criteria Radiographics 1988;487-506.

[5]. Asymptolmatic carotid Atherosclerosis Study (ACAC) Interim Results, NEJM 1999;1163-75.

[6]. Magyar $\mathrm{T}$ et al Extreme hyperliplidemia is associated with increased IMT of CCA in patients below 55 years. European Journal of Surgery 9 (suppl-2): 105-161.

[7]. Wolf PA, D' Agostino RB, Bilanger AJ, Hannel WD. Probability of stroke- A risk profile from the Framingham heart study.Stroke.1991;22:312-318.

[8]. Paivansalo M, Leionomen S, Turunen J, TikkakoskiT,Suramo I et al Quantification of carotid artery stenosis with various Doppler velocity parametres. Radiology 1996;164:108-113.

[9]. Dhamija RK, Dhamija SB. Prevalence of stroke in the rural community-An overview of Indian experience. JAPI . I1998;46(4):351-354.

[10]. Rajagopal KV, Lakhkar BN, Banavali S, Singh NK. Pictorial essay-colour duplex evaluation of carotid occlusive lesions: Ind J RadiolImag 2000.

[11]. SK SETHI, RS SOLANKI, H GUPTA. Colour And Duplex Doppler Imaging Evaluation of Extracranial Carotid Artery In Patients Presenting With Transient Ischaemin Attack And Stroke- A Clinical And Radiological Correlation. Ind J RadiolImag 2005 15:1:9198106:

[12]. Steinke W, Kloetzch C, Hennirici M. Carotid artery disease assessed by colour Doppler flow imaging- correlation with standard Doppler sonography and angiography. A106.

[13]. Erikson SJ, Lawson TL, Middleton WD, Quiroz FA, Macrandersj, Dennisfoley W. Stenosis of internal carotid artery assessement using colour Doppler imaging compared with angiography. AJR 1989;152:1299-1305.

[14]. Middleton WD, Foley WD, Lawson TL. Flow reversal in the normal carotid bifurcation: Colour Doppler flow imaging analysis. Radiology, 1988;167:207-210.

[15]. JS Jeng MY Chung, PK Yip, BS Hwang and YC Chang. Extracranial carotid atherosclerosis and vascular risk factors in different types of ischemic stroke in Taiwan Stroke,25,1989-1993.

[16]. Abbreviations:

[17]. CVA - Cerebrovascular accident, CCA- common carotid artery, ICA- internal carotid artery, PSV-peak systolic volume, EDV- end diastolic volume 